\title{
Prevenção da obesidade de forma multidisciplinar no contexto escolar*
}

\section{Preventing obesity in a multidisciplinary way in the school context}

\author{
Rayana Silva Esmeraldo ${ }^{1}$ \\ Alessandro de Oliveira Silva ${ }^{2}$ \\ Filipe Dinato de Lima ${ }^{3}$ \\ Vitor Vicente da Silva ${ }^{4}$ \\ Renata Aparecida Elias Dantas ${ }^{5}$ \\ Ricardo Jacó de Oliveira ${ }^{6}$ \\ Hugo Alves de Souza ${ }^{7}$ \\ Márcio Rabelo Mota ${ }^{8}$
}

* Recebido em: 09/11/2013 Aprovado em: 04/06/2014

1 Professora de Educação Física graduada no Centro Universitário de Brasília (UniCEUB).

2 Docente do curso de Educação Física do UniCEUB.

3 Professor de Educação Física graduada no Centro Universitário de Brasília (UniCEUB). E-mail: filipedinato@hotmail.com

4 Graduando do curso de Educação Física do UniCEUB.

5 Docente do curso de Educação Física do UniCEUB.

6 Docente da Faculdade de Educação Física da UnB.

7 Docente do curso de Fisioterapia do UniCEUB.

8 Docente do curso de Educação Física do UniCEUB.

\section{Resumo}

O ambiente escolar é ideal ao desenvolvimento e produção de hábitos saudáveis. A aula de educação física escolar (EFE) é um momento propício para exposição de padrões alimentares e físicos adequados, porém não se está aproveitando essa disciplina satisfatoriamente, com menos sistematização dos projetos de ensino e menos deslocamentos durante as aulas. A obesidade nos jovens já é considerada uma epidemia que, se não tratada, aumentam as chances de desenvolvimento de diversas doenças, como diabetes, hipertensão arterial e a síndrome metabólica. O objetivo deste estudo foi descrever métodos que possam contribuir para a prevenção da obesidade nas escolas, por meio da EFE. Para isso, foi realizada uma revisão bibliográfica de artigos, livros e sites, com publicações de 1979, e 2001 a 2012. Na prevenção desta doença, ensaios de base familiar, de longa duração, com treinos combinados e intervenções na dieta e nos exercícios mostraram resultados positivos.

Palavras-chave: Educação física escolar. Obesidade. Tratamento multidisciplinar. Reeducação alimentar. Exercício físico.

\begin{abstract}
School environment is ideal for the development and production of healthy habits. The class of physical education (EFE) is a propitious time for exposing dietary patterns and appropriate physical, but is not currently taking advantage of this course satisfactorily, with fewer systematization of teaching projects and fewer activities during class. Obesity in young people is already considered an epidemic that, if not treated, increase the chances of developing various diseases such as diabetes, hypertension and metabolic syndrome. The aim of this study was to describe methods that can contribute to obesity prevention in schools through the EFE. For this, was performed a literature review of articles and books, with publications from 1979, and 2001 to 2012. In the prevention of this disease, family-based tests of long duration, combined with training (aerobic and resistance) to dietary interventions and exercise showed positive results.
\end{abstract}

Keywords: School physical education. Obesity. Treatment. Food reeducation. Physical exercise. 


\section{Introdução}

A legislação brasileira trata da educação física escolar na Lei de Diretrizes e Bases da Educação - LDB (1996) como componente obrigatório no currículo da educação básica, devendo ser integrado à proposta pedagógica da escola e promovendo práticas desportivas formais e não formais dentro do ambiente educacional.

A escola é um ambiente propício ao desenvolvimento e estimulação de ações na área da saúde, pois é um centro de aprendizagem que observa o aluno dentro de seu contexto (social, familiar, comunitário). É um local onde os estudantes passam boa parte do dia, facilitando a promoção e divulgação de hábitos saudáveis (SILVEIRA et al., 2011; GUEDES et al., 2009).

A Educação Física Escolar (EFE) tem seu papel fundamental para favorecer uma vida mais ativa na infância e adolescência, incentivando estilos de vida saudáveis na fase adulta, pois a inatividade física aumenta os riscos de aparecimento de muitas outras doenças além da obesidade, como as debilidades cardiovasculares, a osteoporose e a diabetes (SILVA, 2008).

De acordo com Guedes e Guedes (2001), as aulas de EFE não estão com intensidades e durações necessárias ao desenvolvimento da aptidão física em alunos. Em contrapartida, as horas em frente à televisão e os hábitos não saudáveis estão aumentando na população estudantil. Em uma realidade mais preocupante, ainda há uma grande quantidade de alunos que, em desconformidade com a LDB, não possuem aulas de EFE em suas instituições de ensino (RIVERA et al., 2010).

Segundo a Pesquisa de Orçamentos Familiares 2008-2009 (IBGE, 2010), 50,1\% dos homens e 48,0\% das mulheres estão com excesso de peso no Brasil. A frequência em crianças entre cinco e nove anos foi de $33,5 \%$ e em adolescentes foi de 20,5\%. A proporção de obesidade cresce a cada ano, chegando a 4,9\% em adolescentes (10-19 anos), 14,8\% em adultos (com mais de 20 anos) e 14,3\% em crianças (5-9 anos), confirmando uma tendência do aumento acelerado do problema. O excesso de peso e a obesidade já são considerados um problema de saúde pública, encontradas com frequência em todas as faixas de renda e regiões do país, em crianças de cinco a nove anos.

O padrão alimentar e de atividade física também foi modificado nos últimos anos, o que explica os números de excesso de peso e obesidade no Brasil (IBGE, salsicha, mortadela, sanduíches e salgados destacaram-se com alta frequência, assim como uma menor ingestão de feijão, saladas e verduras. Em uma análise geral, o biscoito recheado liderou nos marcadores de consumo não saudável (IBGE, 2011). Cerca de 28,2 milhões de pessoas (20,0\% da população brasileira de 14 anos ou mais de idade) não praticam atividades físicas relacionadas à definição de "pessoas ativas" pela OMS (Organização Mundial da Saúde), segundo a Pesquisa Nacional por Amostras de Domicílios - PNAD, realizada em 2008 (IBGE, 2010).

Ainda de acordo com a PNAD 2008 (IBGE, 2010), 28,2\% da população do Brasil, com 14 anos ou mais de idade (41,4 milhões), praticavam exercícios físicos ou esportes. Daqueles que ganhavam menos de um salário mínimo, apenas $20,2 \%$ praticavam exercício físico ou esporte. Dos que ganhavam mais de cinco salários mínimos, o percentual ultrapassava os $50 \%$. Apesar desses 41,4 milhões de pessoas terem declarado que praticam alguma atividade física ou esporte, apenas 10,2\% (ou 14,9 milhões) foram consideradas "ativas no lazer", segundo os critérios da OMS.

A diferença entre o número de células adiposas de obesos e magros se estabelece na infância e, durante a vida adulta, o número total dessas células permanece o mesmo por toda a vida, sendo a obesidade causada pela hiperplasia das células adiposas (KNITTLE et al., 1979). Apenas 10\% das crianças eutróficas passam a desenvolver a obesidade futuramente e aproximadamente $80 \%$ das crianças obesas no presente permanecerão obesas na fase adulta (FREEDMAN et al., 2001). Esses fatores contribuem para que pessoas com obesidade tenham dificuldade para perder peso (SPALDING et al., 2008).

Em virtude da prevalência do início da obesidade nas crianças, faz-se necessária a prevenção dessa doença na escola. Desse modo, o objetivo desse estudo foi descrever métodos que possam contribuir para a prevenção da obesidade nas escolas, por meio principalmente da EFE, a partir da revisão da literatura já existente.

\section{Metodologia}

O presente estudo configura-se como uma revisão bibliográfica sistemática. Foram utilizadas as palavras-chave Obesidade Infantil e Educação Física Escolar nas bases de dados PubMed e Scielo para a busca por artigos científicos relacionados à prevenção da obesidade infantil na escola. Foram selecionados 26 
artigos que atendiam aos critérios de inclusão. Como critérios de inclusão, adotou-se a aquisição de publicações a partir de 2001, tendo crianças com obesidade como grupo investigado e artigos que englobassem prevenção e/ou tratamento da obesidade.

\section{Desenvolvimento}

A participação de crianças e adolescentes em atividades físicas é um objetivo que deve ser perseguido para a prevenção da obesidade, evitando que estes se tornem adultos obesos e que tenham uma vida menos saudável que a dos pais (HILLS; ANDERSEN; BYRNE, 2011). Atuando de forma ativa para prevenir o aumento da obesidade infantil, a saúde pública, o governo, a escola, as indústrias de alimentação e os profissionais da saúde são um conjunto importante para a busca desse objetivo (SOUSA; LOUREIRO; CARMO, 2008).

Segundo a revisão de dados de Neves et al. (2010), é importante uma reeducação alimentar em família, além do tratamento da obesidade infantil com uma equipe multiprofissional, formada por nutricionista, professor de educação física, psicólogo e pediatra, assim a criança reproduzirá a necessária modificação no estilo de vida ajustando sua dieta, a prática de exercícios físicos, o comportamento psicossocial e familiar.

Os dados comparativos analisados a partir de 7 artigos, englobando a prevenção e o tratamento da obesidade, estão expressos na Tabela 1.

Tabela 1 - Propostas para a prevenção e tratamento da obesidade

\begin{tabular}{|c|c|c|c|}
\hline Referência & Amostra & Métodos & Resultados \\
\hline $\begin{array}{l}\text { Coleman et } \\
\text { al. (2005) }\end{array}$ & $\begin{array}{l}896 \text { crianças ( } 93 \% \\
\text { hispânicos), } 423 \text { crianças } \\
\text { testadas e } 473 \text { do grupo } \\
\text { controle, acompanhadas } \\
\text { na quarta e quinta séries. } \\
\text { Estudo com avaliação de } \\
\text { dois anos. }\end{array}$ & $\begin{array}{l}\text { Avaliação inicial do risco de sobrepeso e obesidade, antropometria, } \\
\text { condicionamento aeróbico (corrida de nove minutos), aula de educação física } \\
\text { escolar (intensidade), alimentação (qualidade). Foram treinados os professores } \\
\text { de educação física e pessoas do serviço alimentar para adequações às propostas } \\
\text { do estudo, além de aquisições de materiais para as atividades físicas. } 0 \text { grupo } \\
\text { controle não recebeu nenhum treinamento ou material. Os dois grupos } \\
\text { receberam incentivo financeiro para a participação no programa, porém o grupo } \\
\text { controle não foi orientado sobre como gastar o dinheiro. }\end{array}$ & $\begin{array}{l}\text { A taxa de aumento do risco de sobrepeso para meninas } \\
\text { nas escolas testadas foi significativamente menor } \\
\text { (2\%) em comparação com a taxa do grupo controle } \\
\text { para as meninas ( } 13 \% \text { ). Um padrão semelhante foi } \\
\text { observado para os meninos, com uma taxa de aumento } \\
\text { nas escolas testadas de } 1 \% \text { ( } 40 \% \text { para } 41 \% \text { ), o que foi } \\
\text { significativamente inferior ao aumento de } 9 \% \text { ( } 40 \% \text { a } \\
49 \% \text { ) para meninos do grupo controle. }\end{array}$ \\
\hline $\begin{array}{l}\text { Farias et al. } \\
(2009)\end{array}$ & $\begin{array}{l}383 \text { alunos, divididos em } \\
\text { dois grupos: caso com } 186 \\
\text { ( } 96 \text { meninos e } 90 \text { meninas) } \\
\text { e controle com } 197 \text { ( } 108 \\
\text { meninos e } 89 \text { meninas), } \\
\text { com idade entre } 10 \text { e } 15 \\
\text { anos. Avaliados por um } \\
\text { ano letivo. }\end{array}$ & $\begin{array}{l}\text { Ambos os grupos foram submetidos a duas aulas de educação física semanais, } \\
\text { com duração de } 60 \text { minutos cada sessão e a } 68 \text { aulas anuais. Os escolares do } \\
\text { grupo-controle realizaram atividade física considerada habitual na escola, } \\
\text { como recreação e jogos por meio de brincadeiras, exercícios de calistenia, } \\
\text { aprendizagem de fundamentos das modalidades esportivas e jogos esportivos. } \\
\text { Os escolares do grupo caso foram submetidos à atividade física programada, } \\
\text { sendo monitorizada a frequência cardíaca máxima, com intensidade inicial de } \\
40 \text { a } 55 \% \text { da frequência cardíaca máxima, durante um período máximo de } 1 / 3 \text { do } \\
\text { estudo, intensificando posteriormente para } 55 \text { a } 75 \% \text {. As aulas foram compostas } \\
\text { de três partes: a primeira, com atividade aeróbia (exercícios de flexibilidade, } \\
\text { pular corda, caminhadas, corridas alternadas, saltos em ritmo contínuo e jogos } \\
\text { recreativos) com duração de } 30 \text { minutos; a segunda, com jogos esportivos } \\
\text { (voleibol, futebol de salão, handebol e natação) com duração de } 20 \text { minutos; e } \\
\text { a terceira, com alongamento, com duração de } 10 \text { minutos. }\end{array}$ & $\begin{array}{l}\text { A atividade física programada resultou em melhoria } \\
\text { e manutenção nas variáveis da composição corporal } \\
\text { e redução da frequência de sobrepeso e obesidade } \\
\text { no grupo que sofreu intervenção. Também mostrou a } \\
\text { diminuição significativa da frequência de obesidade } \\
\text { no grupo caso (e não no grupo-controle) no pós-teste } \\
\text { em relação ao pré-teste. A atividade física voltada } \\
\text { para promoção da saúde, realizada durante a atividade } \\
\text { escolar, se não promover uma redução significativa da } \\
\text { gordura corporal, durante o período de aderência da } \\
\text { atividade física, aomenos previne seu aumento, inclusive } \\
\text { na puberdade. }\end{array}$ \\
\hline $\begin{array}{l}\text { Foschini et al. } \\
(2010)\end{array}$ & $\begin{array}{l}\text { Um total de } 32 \\
\text { adolescentes obesos pós- } \\
\text { puberais foram submetidos } \\
\text { a treinamento aeróbio (TA) } \\
\text { e treinamento resistido (TR) } \\
\text { durante } 14 \text { semanas, com } \\
\text { adaptação idêntica para os } \\
\text { dois grupos por } 2 \text { semanas. }\end{array}$ & $\begin{array}{l}\text { Os indivíduos foram divididos em periodização linear (PL, } n=16 \text { ) ou periodização } \\
\text { ondulatória diária (POD, } n=16 \text { ). Consistiu em intervenção multidisciplinar de } \\
\text { obesidade, com aeróbio (AE) + treino de resistência (TR), clínica, nutricional e } \\
\text { terapia psicológica. Todos os indivíduos realizaram três sessões de treino por } \\
\text { semana, consistindo de uma terapia de exercícios combinados (AE + TR). A } \\
\text { duração da AE foi de } 30 \mathrm{~min} \text { / sessão. } 0 \text { modo de exercício foi a corrida em uma } \\
\text { esteira motorizada Os exercícios foram realizados na intensidade da frequência } \\
\text { cardíaca do limiar ventilatório. Os fisiologistas controlaram a frequência cardíaca, } \\
\text { em intervalos de } 5 \text { minutos durante todas as sessões de treinamento. } \\
\text { TR. A duração do TR foi de cerca de } 30 \text { min / sessão. } 0 \text { treinamento foi dividido } \\
\text { seguindo as recomendações do ACSM. Todas as sessões foram supervisionadas } \\
\text { individualmente por um experiente profissional TR. }\end{array}$ & $\begin{array}{l}\text { Foi concluído que não houve diferença estatística entre } \\
\text { PL e grupos POD em todos os parâmetros analisados, } \\
\text { mostrando que a intervenção multidisciplinar foi o fator } \\
\text { determinante para a melhora dos parâmetros de saúde } \\
\text { analisados em adolescentes obesos, submetidos a } 14 \\
\text { semanas de estudo. Ambos os grupos demonstraram } \\
\text { uma redução significativa na massa corporal, IMC, } \\
\text { gordura corporal, visceral e subcutânea, gordura total } \\
\text { e colesterol (lipoproteína de baixa densidade - LDL), } \\
\text { pressão arterial e aumento da massa livre de gordura, } \\
\text { V02máximo, e resistência muscular. Apenas POD } \\
\text { promoveu uma redução nas concentrações de insulina } \\
\text { e HOMA-IR. }\end{array}$ \\
\hline
\end{tabular}




\begin{tabular}{|c|c|c|}
\hline $\begin{array}{l}\text { Togashi, } \\
\text { Masuda e } \\
\text { Iguchi (2010) }\end{array}$ & $\begin{array}{l}\text { Os sujeitos foram } 33 \\
\text { crianças ( } 23 \text { meninos e } 10 \\
\text { meninas) com idade média } \\
\text { de } 10,1(7-14 \text { anos) com } \\
\text { um percentual de gordura } \\
\text { corporal de } 37,3 \% \text {. Foram } \\
\text { internados para tratamento } \\
\text { de } 3 \text { meses enquanto } \\
\text { participavam de uma escola } \\
\text { ligada ao hospital. }\end{array}$ & $\begin{array}{l}\text { As áreas de gordura subcutânea e visceral foram medidas. Amostras de sangue } \\
\text { foram tomadas para medir os fatores de risco para doença crônica, como os tri- } \\
\text { glicerídeos (TG), colesterol total (T-CH0), lipoproteína de alta densidade (HDL), } \\
\text { insulina, asparato amino-transferase (AST), alanina aminotransferase (ALT) e } \\
\text { ácido úrico (AU). Tratamento de exercício supervisionado no V02max de } 50 \% \\
\text { para cerca de } 60 \mathrm{~min} \text { / dia foi realizado } 5 \text { ou } 7 \text { dias por semana durante } 3 \text { meses. } \\
0 \text { tratamento dietético foi variado com ingestão diária entre } 1400 \text { e } 1900 \mathrm{kcal,} \\
\text { dependendo do grau de obesidade. No tratamento com exercício, os sujeitos pra- } \\
\text { ticaram ginástica de rádio e uma caminhada de } 15 \text { minutos pela manhã, exer- } \\
\text { cício no step por volta de } 20 \text { minutos usando um haltere leve e plataforma de } \\
10 \text { cm de altura e um exercício diário de resistência (10 minutos), utilizando sua } \\
\text { próprio peso corporal durante a noite com o enfermeiro, como uma regra. Além } \\
\text { disso, eles praticavam basquetebol, uma vez por semana e aeróbica duas vezes } \\
\text { por semana durante cerca de } 1 \text { hora após o jantar. A duração da intervenção foi } \\
\text { de } 3 \text { meses. A intensidade de exercício foi ajustada para o ritmo cardíaco indivi- } \\
\text { dual dos sujeitos, em } 50 \% \text { do V02max. }\end{array}$ \\
\hline $\begin{array}{l}\text { Mello et al. } \\
\text { (2011) }\end{array}$ & $\begin{array}{l}\text { Os sujeitos eram } 30 \\
\text { pacientes adolescentes } \\
\text { pós-puberais, sendo } 16 \\
\text { adolescentes obesos (índice } \\
\text { de massa corporal [IMC] } \\
\text { maior que o percentil } 95 \text { ), } \\
17 \text { que tinham entre } 15 \text { e } \\
19 \text { anos de idade (16,71 } \\
\pm 1,47 \text { anos). Eram } 10 \\
\text { meninas e } 20 \text { meninos que } \\
\text { foram recrutados para um } \\
\text { estudo de longo prazo de } \\
\text { intervenção (um ano). }\end{array}$ & $\begin{array}{l}\text { Os participantes foram divididos em dois grupos: um ano de treino aeróbio (TA)e } \\
\text { treino resistido (TR), TA + TR: } n=15 \text { ou um ano apenas de treino aeróbio (TA), TA: } \\
n=15 \text {. Antes do início do estudo, ambos os grupos realizaram } 2 \text { semanas de trei- } \\
\text { namento similar para adaptação. Todos os pacientes relataram que não tinham } \\
\text { nenhuma experiência com TR antes do estudo. As avaliações foram realizadas } \\
\text { no início, após } 6 \text { meses (curto prazo) e depois de } 1 \text { ano (longo prazo) da terapia } \\
\text { interdisciplinar. Todos os participantes foram completamente familiarizados com } \\
\text { todos os procedimentos de ensaio antes da experiência para reduzir a influência } \\
\text { de quaisquer efeitos de aprendizagem. Atendimento multidisciplinar com tera- } \\
\text { pia clínica, nutricional e psicológica, além do exercício físico. }\end{array}$ \\
\hline $\begin{array}{l}\text { Spiegel e } \\
\text { Foulk (2006) }\end{array}$ & $\begin{array}{l}1013 \text { estudantes de } \\
\text { quarta e quinta séries de } \\
69 \text { classes, em } 16 \text { escolas } \\
\text { diferentes. As turmas } \\
\text { de intervenção e grupo } \\
\text { controle foram selecionadas } \\
\text { aleatoriamente. } \\
\text { Intervenção por um ano } \\
\text { letivo. }\end{array}$ & $\begin{array}{l}\text { Projeto multiprofissional envolvendo a escola, a nutrição, os pais, além de en- } \\
\text { volver a interdisciplinariedade (educação física, leitura, escrita, matemática, } \\
\text { ciências e estudos sociais). } 35 \text { professores foram convidados para participar do } \\
\text { grupo intervenção e } 35 \text { para o grupo controle. Professores do grupo intervenção } \\
\text { participaram de oficinas sobre o projeto de atuação e receberam materiais do } \\
\text { programa. }\end{array}$ \\
\hline $\begin{array}{l}\text { Telford et al. } \\
\text { (2012) }\end{array}$ & $\begin{array}{l}\text { Estudo longitudinal de dois } \\
\text { anos que envolveu } 620 \\
\text { meninos e meninas. }\end{array}$ & $\begin{array}{l}\text { As medições foram porcentagem de gordura corporal, escrita, matemática e } \\
\text { proficiência de leitura (por testes do governo). } 0 \text { estudo foi realizado com profes- } \\
\text { sores especialistas para a pesquisa (ensinou EF para } 312 \text { alunos, por } 90 \text { minutos/ } \\
\text { semana) e um grupo controle com os professores de sala de aula que continu- } \\
\text { aram ensinando programas comumente praticados EF (prática comum EF para } \\
308 \text { alunos, de acordo com a prática habitual). Um grupo controle sem EF não } \\
\text { é eticamente aceitável, por isso foi calculado } 0 \text { efeito do professor especialista } \\
\text { em referência ao efeito da prática comum EF. Foram } 29 \text { escolas participantes do } \\
\text { estudo. }\end{array}$ \\
\hline
\end{tabular}

Após o tratamento combinado com tratamento dietético e exercício, as áreas de gordura subcutânea e visceral diminuíram significativamente e fatores de risco tais como TG, T-CHO, insulina, AST, ALT e AU foram visivelmente reduzidas. Estes dados sugerem que 0 tratamento combinado com tratamento dietético e exercício em crianças obesas normaliza a distribuição da gordura abdominal e reduz os fatores de risco para doenças crônicas.

Os resultados do presente estudo demonstraram que TA + TR eram mais eficazes do que o TA sozinho no controle da síndrome metabólica em adolescentes obesos após longo prazo de intervenção. De fato, o TA + TR proporcionou uma melhoria contínua de vários parâmetros a partir de 6 meses a 1 ano de intervenção, incluindo IMC, massa gorda, a gordura visceral, glicose, insulina, HOMA-IR, e adiponectinemia.

0 programa demonstrou mudanças positivas no IMC, consumo de frutas e legumes e atividade física. Percebeu-se a capacidade de influenciar transformações, com os dados indicando que é possível reduzir excesso de peso por meio de intervenções escolares e mudanças nos comportamentos familiares.

Em comparação com a prática comum de EF, o especialista ensinou EF foi associado com um menor aumento da porcentagem de gordura corporal relacionada com a idade. Especialista que ensinou EF também foi associado com grandes melhorias em matemática e escrita. Não houve evidência de um efeito de leitura. Crianças do ensino fundamental que receberam PE de especialistas forneceram suporte para o papel daEF, tanto na medicina preventiva quanto no desenvolvimento acadêmico.
Dentro do ambiente escolar, a alimentação saudável e a prática de exercícios físicos estão no contexto das aulas de EFE, podendo ser desenvolvidas para prevenir e controlar a obesidade em crianças e adolescentes. Porém, as aulas de EFE estão se apresentando pouco sistematizadas, com menos deslocamentos e sem progressão do conhecimento. Essa negligência dentro das aulas está desassociando um componente essencial da educação física, o movimento (ARAÚJO; BRITO; SILVA, 2010).

A comunidade e a família têm um papel fundamental na distribuição de valores voltados para esse movimento, intervindo assim na saúde. A escola atua primariamente influenciando os níveis de educação alimentar e o gosto pela prática de exercícios físicos, criando condições para serem colocados em prática pela sociedade (COELHO et al., 2008). Segundo McCall e Raj (2009), alguns estudos de base familiar têm-se mostrado eficazes na prevenção da obesidade, apesar de a maioria dos ensaios serem escolares, pois são mais fáceis de planejar e executar. O estudo de Spiegel e Foulk (2006) demonstra que esse tipo de intervenção pode dar resultados satisfatórios.

Ainda conforme McCall e Raj (2009), as intervenções escolares, em conjunto com a família, podem estimular a extensão das atividades para o período noturno pela comunidade, beneficiando adolescentes em tran- 
sição para a fase adulta e estimulando continuamente a prática de exercícios. Componentes ambientais, comportamentais e educacionais são uma importante combinação na obtenção de resultados positivos.

Desse modo, os programas de intervenção que envolvam as famílias se mostram mais eficazes no tratamento de crianças com excesso de peso e obesidade, assim como intervenções mais longas, com duração superior a um ano (SICHIERI; SOUZA, 2008). Nesse sentido, Telford et al. (2012) encontraram resultados positivos em seu estudo, no qual houve um acompanhamento de dois anos, além de avaliações fisiológicas e análises do desenvolvimento intelectual. Com isso, a escola se mostra como um ambiente propício às intervenções, pois promove uma interação a longo prazo entre os participantes dos programas.

Sendo um componente obrigatório, as aulas de EFE necessitam de programas de intervenção em conjunto com outros profissionais, utilizando a interdisciplinaridade como um método de trabalho (MARTÍNEZ et al., 2012), como Foschini et al. (2010) atribuem os resultados positivos de seu estudo. A comunidade europeia tem procurado projetos emergenciais em busca de uma alimentação balanceada fora de casa em conjunto com empresas alimentícias (SICHIERI; SOUZA, 2008).

Os esforços de emergência para a produção de hábitos mais saudáveis, além do envolvimento das empresas alimentícias, necessitam da escola tanto como um ambiente educativo como um exemplo a ser seguido, como na venda de alimentos saudáveis a serem disponibilizados na instituição (SICHIERI; SOUZA, 2008). Ações como o aproveitamento dos horários escolares livres, como os intervalos, também podem ser aproveitadas para o estímulo das atividades físicas (COELHO et al., 2008).

Porém, segundo Gutin (2008), apenas mudanças que privilegiem modificações na dieta não são tão eficazes para a prevenção. Os esforços devem ser concentrados na composição corporal, ao invés de peso corporal e restrição de consumo de energia. As atividades físicas devem ser estimuladas, principalmente a atividade física vigorosa. Vários autores concordam que atividades nas aulas de EFE devam ser de intensidade moderada a vigorosa para uma intervenção com melhores resultados (MARTÍNEZ, 2012; GUTIN, 2011; HILLS; BYRNE, 2006; MCCALL; RAJ, 2009; KIM, 2012).

Mello et al. (2011) realizaram um estudo que comparou os treinos aeróbicos com treinos mistos (ae- róbio e resistência) para verificar qual é o mais efetivo na melhora da síndrome metabólica e da adiponectinemia em adolescentes obesos. Durante o período de um ano, acompanharam esses adolescentes e ao final do estudo, o treino misto com aeróbio e resistência apresentou melhores resultados. Assim, é importante acrescentar essas características às aulas de EFE, além das intensidades moderadas a vigorosas, buscando a realização de aulas com melhores projetos e mais eficazes.

Em um estudo com alunos coreanos, Wi-Young et al. (2011) concluíram que a frequência das aulas de EFE tem papel importante para uma melhora no peso dos estudantes, sendo uma ferramenta para prevenção da obesidade. Foi encontrada, em consonância com outros estudos, maior prevalência de sobrepeso e obesidade nas meninas do que em meninos. Porém, uma hora de aula de educação física é mais efetiva na redução de peso em meninas do que em meninos. Esses dados corroboram os resultados apresentados por Coleman et al. (2005), mais expressivos nas meninas.

No estudo realizado por Martínez et al. (2012) com crianças de 11 e 12 anos na cidade de Toledo (Espanha), após a análise dos dados de acelerômetros, apenas $22,2 \%$ das crianças avaliadas atendiam às recomendações internacionais do mínimo de 60 minutos de atividade física de intensidade moderada a vigorosa por dia, sendo os meninos mais ativos e com maiores intensidades em suas atividades. Na pesquisa de Celestrino e Costa (2006), as meninas com sobrepeso ou obesidade possuíam uma predominância de atividades sedentárias (como assistir TV e fazer lição de casa) e os meninos possuíam um período prolongado utilizando equipamentos eletrônicos (como TV e videogames).

Os ambientes para as aulas de educação física devem ser expandidos para outros momentos fora do horário escolar, como as férias, fins de semana e horários noturnos. Atividades vigorosas, como corridas, basquete, natação e dança, possuem maior estimulação mecânica dos tecidos. Para crianças com sobrepeso ou obesidade, deve-se começar gradativamente com cargas mais baixas de volume e intensidade, a fim de evitar fadiga exagerada ou lesão (GUTIN, 2008).

Assim, é importante que as atividades moderadas a vigorosas sejam executadas pelas crianças de forma voluntária, pois segundo a revisão sistemática de Connellya, Duasob e Butlerc (2007), entre 28 casos eleitos para o estudo, 17 foram identificados como ineficazes na redução 
da adiposidade. Segundo os autores, o principal fator que diferenciou os estudos foram as atividades realizadas de maneira obrigatória pelos sujeitos. Gutin (2008) comenta que as atividades devam ser adequadas para as diferentes idades, pois os jogos são preferidos por crianças mais jovens, enquanto aparelhos de musculação já são bem aceitos pelos adolescentes.

Um fator que pode desestimular a participação de populações alvo (crianças com sobrepeso e obesidade) nas aulas de educação física são as atividades propostas pelos professores. Atividades que geram deslocamentos corporais ou acentuem a dificuldade motora afastam esses alunos das aulas (CELESTRINO; COSTA, 2006). Cabe ao profissional responsável pela EFE oferecer atividades que não afugentem os alunos e que ao mesmo tempo os estimulem fisicamente de maneira satisfatória.

Em uma meta-análise, Harris et al. (2009) afirmam que as intervenções possuem menos participação de crianças com IMC elevado, consequentemente a baixa adesão leva a um menor beneficiamento dessa população nos programas de intervenção. Além disso, podem não influenciar a composição corporal pela ingestão dietética elevada. Os autores concluem que os estudos avaliados não melhoraram o IMC a curto e longo prazo, porém analisam que as cargas das intensidades e volumes em aulas de EFE devem ser melhor avaliadas para futuros estudos, pois o ambiente escolar é um importante cenário para as intervenções, devendo incluir múltiplas modificações, como na melhoria da dieta, que mostram-se promissoras nos estudos.

No estudo de Togashi, Masuda e Iguchi (2010), verificou-se uma diminuição nos resultados da gordura subcutânea e visceral, além da diminuição significativa dos triglicerídeos, colesterol, insulina, asparato amino-transferase, alanina aminotransferase e ácido úrico, após intervenção dietética e no exercício físico. Observou-se, também, uma melhor distribuição da gordura abdominal e redução dos fatores de risco para doenças crônicas. Porém, o estudo foi realizado dentro de um hospital, onde há um controle maior, na internação, das variáveis da pesquisa. Os resultados são expressivos, entretanto dificultam sua aplicação a grandes grupos escolares, mas a pesquisa é relevante justamente por mostrar que mudanças na dieta e no aumento da atividade física podem dar resultados positivos.

Os atuais relatórios indicam uma maior partici- pação das crianças nos desportos organizados e na EFE, porém as atividades físicas incidentais, como transporte ativo para a escola, caminhando, diminuiu. A literatura adverte que crianças de vários países não são suficientemente ativas, concordando com a tendência do aumento do sobrepeso e da obesidade, menor desempenho físico e baixa aptidão. Evidências indicam que a saúde das crianças ocidentais tem se deteriorado nas últimas décadas (HILLS; ANDERSEN; BYRNE, 2011).

Segundo estudo americano, em uma estimativa por baixo, a cada 6 dólares gastos com cuidados à saúde, 1 dólar seria atribuído aos custos com excesso de peso e obesidade. Apesar das estimativas e proporções possam ser afetadas por políticas públicas de saúde, nos EUA, segundo a literatura, não há sinais significativos de que o aumento da obesidade irá atenuar. Programas eficazes contra o sobrepeso e a obesidade podem levar anos para apresentar resultados expressivos. A implementação de programas e políticas públicas eficientes são necessárias para o enfrentamento da epidemia, como afirmam a OMS e o Instituto de Medicina com relação à obesidade infantil nos EUA (WANG et al., 2008).

Apesar de a maioria dos estudos não mostrarem uma diminuição do IMC, diversos estudos têm demonstrado uma redução significativa no percentual de gordura, além de melhores marcadores de resistência à insulina e da inflamação por meio dos exercícios físicos. Os benefícios do exercício se estendem para além da perda de peso. As diferenciadas intervenções, de acordo com as idades, meio ambiente e fatores de risco são necessárias, ao invés de priorizar uma única intervenção (MCCALL; RAJ 2009). Farias et al. (2009) comentam que se a aula de EFE não apresentar uma redução significativa de gordura corporal, ao menos previne seu aumento.

Wofford (2008) apresentou uma revisão sistemática sobre a prevenção da obesidade infantil e relatou que em uma pesquisa com mais de 300 médicos, $71 \%$ da amostra se sentem incapazes de tratar a obesidade. Esse dado demonstra as dificuldades que os profissionais da saúde sentem ao tratar a obesidade, pois a etiologia não é única.

Assim, os governos e as entidades devem se unir às famílias e comunidades no enfrentamento dessa epidemia, com projetos e programas de saúde que encarem o problema global. Muito ainda precisa ser feito para que providências úteis sejam implementadas, porém já se 
observa alguns projetos específicos, como o Programa Saúde na Escola (PSE), instituído em 2007 pelo Decreto Presidencial no 6.286 e lançado em parceria pelos Ministérios da Saúde e Educação, em setembro de 2008, com as diretrizes apresentadas em 2011. O programa abordará diversos níveis de intervenção, envolvendo principalmente as escolas públicas brasileiras (MINISTÉRIO DA SAÚDE, 2011).

\section{Conclusões}

Conclui-se que a EFE é um importante meio de utilização para a prevenção da obesidade de escolares brasileiros, devendo ter sua estruturação e sistematização dos projetos aprimorada e, consequentemente, tornar-se efetiva e ativa nas escolas. As aulas devem ser programadas com ludicidade, tendo intensidades moderadas a vigorosas, com treinos aeróbios e de resistência. O tempo médio nos estudos apresentam aulas ou treinos de $60 \mathrm{mi}-$ nutos. Os benefícios encontrados nos estudos utilizados neste artigo indicam melhoras fisiológicas, psicológicas (humor) e acadêmicas (matemática e escrita). É importante a abertura das escolas para a prática de exercícios físicos em horários distintos das aulas, como os intervalos, as férias, os finais de semana e os períodos noturnos, com a participação da comunidade. As instituições de ensino devem trabalhar seus conteúdos curriculares envolvendo essa dinâmica, além de capacitar a comunidade e as famílias para hábitos mais saudáveis. As famílias necessitam participar em conjunto com o tratamento das crianças com sobrepeso e obesidade, além do tratamento ser multiprofissional, envolvendo educador físico, pediatra, nutricionista, psicólogo, pois a obesidade é uma doença multifatorial.

A prevenção da obesidade acaba por prevenir também o aparecimento de outras doenças, auxiliando a manutenção de um estilo de vida saudável, com tendência a se estender pela vida adulta, podendo diminuir os custos da saúde pública. Dessa forma, o fato de existirem escolas que não ofereçam aulas de EFE em seus currículos representa um grande desafio.

Os estudos apresentados neste artigo apresentam intervenções para tratamento da obesidade, porém são admitidos utilizá-los na prevenção da obesidade escolar por atuarem de forma semelhante fisiologicamente. $\mathrm{O}$ tratamento dessa doença requer muita atenção e cuidado dos profissionais de saúde, principalmente pelos múltiplos fatores que essa doença pode influenciar.

\section{Referências}

ARAÚJO, R. A.; BRITO, A. A.; SILVA, F. M. O papel da educação física escolar diante da epidemia da obesidade em crianças e adolescentes. Educação Física em Revista, Taguatinga, v.4, n. 2, maio/ ago. 2010.

BRASIL. Lei no 9.394 de 20 de dezembro de 1996. Estabelece as diretrizes e bases da educação nacional. Brasília, 1996. Disponível em: <http://www.planalto.gov.br/ ccivil_03/LEIS/19394.htm>. Acesso em: 02 abr. 2012.

BRASIL. Ministério da Saúde. Secretaria de Atenção à Saúde. Programa saúde na escola: tecendo caminhos da intersetorialidade. Brasília: Ministério da Saúde, 2011.

CELESTRINO, J. O.; COSTA, A. S. A prática de atividade física entre escolares com sobrepeso e obesidade. Revista Mackenzie de Educação Física e Esporte, São Paulo, v. 5, n. 3, p. 47-54, set./dez. 2006.

COELHO, R. et al. Excesso de peso e obesidade: prevenção na escola. Acta Médica Portuguesa, Lisboa, v. 21, n. 4, p. 341-344, jul./ago. 2008.

COLEMAN, K. J. et al. Prevention of the epidemic increase in child risk of overweight in low-income schools: the el paso coordinated approach to child health. Archives of Pediatrics and Adolescents Medicine, Chicago, v. 159, n. 3, p. 217-224, mar. 2005. doi:10.1001/archpedi.159.3.217

CONNELLYA, J.B.; DUASOB, M.J.; BUTLERC, G. A systematic review of controlled trials of interventions to prevent childhood obesity and overweight: A realistic synthesis of the evidence. Public Health, London, v. 121, n. 7, p. 510-517, jul. 2007. doi: 10.1016/j.puhe.2006.11.015

FARIAS, E. S. et al. Influence of programmed physical activity on body composition among adolescent students. Jornal de Pediatria, Rio de Janeiro, v.85, n. 1, p. 28-34, jan./fev. 2009. doi: 10.1590/S0021-75572009000100006

FOSCHINI, D. et al. Treatment of obese adolescents: the influence of periodization models and ace genotype. Obesity, Malden, v. 18, n. 4, p. 766-772, apr. 2010. doi: 10.1038/oby.2009.247

FREEDMAN, D. S. et al. Relationship of childhood overweight to coronary heart disease. risk factors in adulthood: the bogalusa heart study. Pediatrics, Springfield, v. 108, n. 3, p. 712-718, sep. 2001. doi: 10.1542/peds.108.3.712

GUEDES, D. P.; GUEDES, J. E. R. P. Esforços físicos nos programas de educação física escolar. Revista Paulista de Educação Física, São Paulo, v. 15, n. 1, p. 33-44, jan/ jun. 2001.

GUEDES, N. G. et al. Atividade física de escolares: análise segundo o modelo teórico de promoção da saúde de Pender. Revista da Escola de Enfermagem da USP, v. 43, n. 4, p. 744-780, dez. 2009. doi: 10.1590/S0080-62342009000400006 
GUTIN, B. Child obesity can be reduced with vigorous activity rather than restriction of energy intake. Obesity, Malden, v. 16, n. 10, p. 2193-2196, oct. 2008. doi: 10.1038/ oby. 2008.348

GUTIN, B. Diet vs exercise for the prevention of pediatric obesity: the role of exercise. International Journal of Obesity, London, v. 35, n. 1. p. 29-32, jan. 2011. doi:10.1038/ijo.2010.140

HARRIS, K. C. et al. Effect of school-based physical activity interventions on body mass index in children: a meta-analysis. CMAJ, Ottawa, v. 180, n. 7, p. 719-726, mar. 2009. doi: $10.1503 /$ cmaj.080966.

HILLS, A. P.; ANDERSEN, L. B.; BYRNE, N. M. Physical activity and obesity in children. British Journal of Sports Medicine, London, v. 45, n. 11, p. 866-870, sep. 2011. doi:10.1136/bjsports-2011-090199

INSTITUTO BRASILEIRO DE GEOGRAFIA E ESTATÍSTICA (IBGE). Pesquisa nacional por amostras de domicílios 2008: um panorama da saúde no Brasil: acesso e utilização dos serviços, condições de saúde e fatores de risco e proteção à saúde. Rio de Janeiro: IBGE, 2010.

INSTITUTO BRASILEIRO DE GEOGRAFIA E ESTATÍSTICA (IBGE). Pesquisa de orçamentos familiares 2008-2009: análise do consumo alimentar pessoal no Brasil. Rio de Janeiro: IBGE, 2011.

KIM, J. Are physical education-related state policies and schools' physical education requirement related to children's physical activity and obesity? Journal of School Health, Columbus, v. 82, n. 6, p. 268-276, jun. 2012. doi: 10.1111/j.1746-1561.2012.00697.x

KNITTLE, J. L. et al. The growth of adipose tissue in children and adolescents. Cross-sectional and longitudinal studies of adipose cell number and size. Journal of Clinical Investigation, New Haven, v. 63, n. 2, p. 239-246, feb. 1979. doi:10.1172/JCI109295

MARTÍNEZ, J. M. et al. Níveis de atividade física medidos com os alunos do acelerômetro in $3^{\circ}$ ciclo educación primária: atividade física diária e sessões de educação física. Revista de Psicología del Deporte, Espanha, v. 21, n. 1, p. 117-123, jan./jun. 2012.

MCCALL, A.; RAJ, R. Exercise for prevention of obesity and diabetes in children and adolescents. Clinics in Sports Medicine, Philadelphia, v. 28, n. 3, p. 393-421, jul. 2009. doi: 10.1016/j.csm.2009.03.001

MELLO, M. T. et al. Long-term effects of aerobic plus resistance training on the metabolic syndrome and adiponectinemia in obese adolescents. Journal of Clinical Hypertension, New York, v. 13, n. 5, p. 343-350, may, 2011. doi: 10.1111/j.1751-7176.2010.00388.x
NEVES, P. M. J. et al. Importância do tratamento e prevenção da obesidade infantil. Educação Física em Revista, v. 4, n. 2, maio/ago. 2010.

RIVERA, I. R. et al. Atividade física, horas de assistência à TV e composição corporal em crianças e adolescentes. Arquivos Brasileiros de Cardiologia, São Paulo, v. 95 , n. 2 , p. $159-165$, ago. 2010. doi: 10.1590/S0066782X2010005000065

SICHIERI, R.; SOUZA, R. A. Estratégias para prevenção da obesidade em crianças e adolescentes. Cadernos de Saúde Pública, Rio de Janeiro, v. 24, p. 209-234, dez. 2008. doi: 10.1590/S0102-311X2008001400002

SILVA, F. M. Educação física escolar e educação para a saúde. Revista Brasileira de Ciências da Saúde, João Pessoa, v. 12, n. 1, p. 81-94, jan./abr. 2008.

SILVEIRA, J. A. C. et al. Effectiveness of school-based nutrition education interventions to prevent and reduce excessive weight gain in children and adolescents: A systematic review. Jornal de Pediatria, Rio de Janeiro, v. 87, n. 5, p. 382-392, set./out. 2011. doi: 10.2223/JPED.2123

SOUSA, J.; LOUREIRO, I.; CARMO, I. A obesidade infantil: um problema emergente. Saude e Tecnologia, Lisboa, v. 1, n. 2, p. 5-15, nov. 2008.

SPALDING, K. L. et al. Dynamics of fat cell turnover in humans. Nature, London, v. 453, n. 7196, p. 783-787, jun. 2008. doi: $10.1038 /$ nature 06902

SPIEGEL, S. A.; FOULK, D. Reducing overweight through a multidisciplinary school-based intervention. Obesity, Malden, v. 14, n. 1, p. 88-96, jan. 2006. doi: 10.1038/oby.2006.11

TELFORD, R. D. et al. Physical education, obesity, and academic achievement: a 2-year longitudinal investigation of australian elementary school children. American Journal of Public Health, New York, v. 102, n. 2, p. 368375, feb. 2012. doi: 10.2105/AJPH.2011.300220

TOGASHI, K.; MASUDA H.; IGUCHI, K. Effect of diet and exercise treatment for obese japanese children on abdominal fat distribution. Research in Sports Medicine: An International Journal, London, v. 18, n. 1, p. 62-70, jan./abr. 2010. doi: 10.1080/15438620903423924

WANG, Y. et al. Will all americans become overweight or obese? estimating the progression and cost of the us obesity epidemic. Obesity, Silver Spring, v. 16, n. 10, p. 2323-2330, oct. 2008. doi: 10.1038/oby.2008.351

WERNECK, F. Z.; NAVARRO, C. A. Nível de atividade física e estado de humor em adolescentes. Psicologia: teoria e pesquisa, Brasília, v. 27, n. 2, p. 189-193, abr./jun. 2011. doi: 10.1590/S0102-37722011000200010 
WI-YOUNG S. et al. Prevalence of obesity in Korean adolescents and its relationship with the weekly frequency of the physical education classes. Journal of Sports Science and Medicine, Turkey, v. 10, n. 4, p. 679-684, dec. 2011.

WOFFORD, L. G. Systematic review of childhood obesity prevention. Journal of Pediatric Nursing, Philadelphia, v. 23, n. 1, fev. 2008. doi: 10.1016/j.pedn.2007.07.006. 